\title{
Influence of melatonin on the testicular regression induced by subcutaneous testosterone pellets in male rats kept in long or short photoperiod
}

\author{
M. K. Vaughan, S. Oaknin*, B. Cozzi†, K. Li $\ddagger$ and R. J. Reiter \\ Department of Cellular and Structural Biology, The University of Texas Health Science Center at \\ San Antonio, San Antonio, Texas 78284, U.S.A.
}

\begin{abstract}
Summary. Daily afternoon injections of $25 \mu \mathrm{g}$ melatonin for 12 weeks had no effect on testicular weights of male rats kept in long photoperiod (14L:10D); similarly, exposure of rats to short photoperiod (2L:22D) had no effect on gonadal weight. However, rats maintained in a long or short photoperiod and implanted every 2 weeks with a $15 \mathrm{~mm}$ Silastic pellet containing testosterone showed a significant reduction in testicular weight; this effect was more pronounced in rats exposed to a short photoperiod. Melatonin injections in testosterone-treated rats in a long photoperiod exacerbated the inhibitory effects of testosterone alone. Subcutaneous 2-weekly implants of a beeswax pellet containing $1 \mathrm{mg}$ melatonin reversed the effects of the melatonin injections on relative testicular weights but not those due to short photoperiod exposure.

Testosterone implants significantly reduced pituitary LH values in long and short photoperiod-exposed animals, more particularly in those exposed to short photoperiod. Melatonin injections alone or in combination with melatonin pellets did not further exaggerate the depression in pituitary LH due to testosterone alone in long photoperiod-exposed animals; similarly melatonin pellets did not reverse the depression in pituitary $\mathrm{LH}$ observed. No significant differences in plasma prolactin concentrations or in thyroxine concentrations or free thyroxine index were observed after any combination of treatments.

We therefore suggest that the effects observed with short photoperiod may be due to melatonin.
\end{abstract}

Keywords: melatonin, testosterone pellets, photoperiod, $\mathrm{LH}$, prolactin, $\mathrm{T}_{4}, \mathrm{~T}_{3}$

\section{Introduction}

The highly inbred laboratory rat presently available from commercial sources is a non-seasonal breeder whose reproductive life history is no longer inextricably tied to natural photoperiodic cues. Nevertheless, Wallen \& Turek (1981) reported that short daylengths (6 h light: $18 \mathrm{~h}$ dark; 6L:18D) could induce a substantial reduction in testicular weight if the animals were simultaneously bearing testosterone-filled Silastic capsules. Since the pineal gland is known to mediate many of the effects of photoperiod, their work suggested that adult male inbred rats still had some components of the pineal-neuroendocrine-reproductive axis that were sensitive to changing daylengths.

\footnotetext{
*Present address: Departamento de Fisiologia y Bioquimica, Universidad de La Laguna, Tenerife, Islas Canarias, Spain.

$\lceil$ Present address: Instituto di Anatomia degli Animali Domestici, Universita degli Studi di Milano, Via Celoria 10, 20122 Milano, Italy.

‡Present address: Department of Anatomy, University of South Carolina, School of Medicine, Columbia, SC 29208, U.S.A.
} 
The indoleamine melatonin is the principal hormone secreted by the pineal and it seems to be responsible for many of the interactions of the gland with the reproductive and thyroid axes (Reiter et al., 1985). In most seasonal breeders tested to date, melatonin is as effective as short photoperiod in eliciting reproductive atrophy. In some species, e.g. the Syrian hamster, melatonin can evoke reproductive atrophy when administered as a daily late afternoon injection. This atrophy is preventable by additional use of short photoperiod or melatonin administered as a chronicallyreleasing pellet; this effect with the pellet has been termed the counter-antigonadotrophic effect of melatonin (Reiter et al., 1974, 1975b). However, in non-seasonal breeders like the rat, the bulk of the evidence suggests that melatonin has an inhibitory action on pubertal development but little effect by itself in the adult (Reiter et al., 1985; Lang, 1986).

Chronic exposure to short photoperiod depresses circulating thyroxine concentrations in hamsters (Mesocricetus auratus) but is ineffective in white-footed mice (Peromyscus leucopus), gerbils (Meriones unguiculatus) or in two strains of mice (Mus musculus, Snell strain and C57BL strain) (Petterborg et al., 1984) and has not been thoroughly evaluated in the rat. As in the reproductive studies mentioned above, melatonin injections late in the light phase are as potent as short photoperiod in inducing a depression in circulating levels of thyroxine in the hamster (Vaughan et al., 1985). In the experiment reported herein, we tested whether injections of melatonin were as effective as short photoperiod in causing hormonal and gonadal weight changes in the testosterone-primed adult male rat; additionally, we evaluated the counter-antigonadotrophic effects of melatonin in rats that were exposed to a short photoperiod and bearing testosterone implants. Finally, we explored the interaction of testosterone, photoperiod and melatonin treatments on the neuroendocrine-thyroid axis.

\section{Materials and Methods}

\section{Preparation of pellets and injections}

Melatonin (Sigma Chemical Co., St Louis, MO) was dissolved in absolute alcohol and maintained as a stock solution at $-10^{\circ} \mathrm{C}$. At the time of injection the stock solution was diluted $1: 10$ with physiological saline $(0 \cdot 9 \mathrm{~g} \mathrm{NaCl} / \mathrm{l})$. Between 16:00 and 18:00 h, the animals in long photoperiod (14L:10D) were given daily subcutaneous injections of $25 \mu \mathrm{g}$ melatonin $/ 0 \cdot 1 \mathrm{ml}$ or the diluent solution.

Melatonin pellets were prepared by mixing $1 \mathrm{mg}$ melatonin in $24 \mathrm{mg}$ softened beeswax; blank pellets without melatonin were prepared for the controls. Testosterone pellets were prepared by filling $15 \mathrm{~mm}$ of Silastic tubing (No. 602-305, Dow Corning, Midland, MI) with crystalline testosterone (Lot No. 103F-0623, Sigma Chemical Co.) and sealing the ends with Type A Silastic medical adhesive; blank capsules were prepared for the controls. The testosterone pellets were soaked overnight in saline $(0 \cdot 9 \mathrm{~g} \mathrm{NaCl} / 1)$ before implantation.

\section{Surgery}

Animals were given implants of blank, testosterone-filled and/or melatonin-filled pellets once every 2 weeks. The animals were anaesthetized with ether and the pellet was inserted subcutaneously on the back.

\section{Experimental protocol}

Young adult male Sprague-Dawley rats (Harlan, Houston, TX; 180-200 g) were acclimatized to constant conditions of photoperiod $(14 \mathrm{~L}: 10 \mathrm{D}$; lights on $06: 00 \mathrm{~h})$ and temperature $\left(20 \pm 2^{\circ} \mathrm{C}\right)$ for 2 weeks. Forty-five animals in a long photoperiod (14L:10D) were separated into 5 groups: (1) diluent only; (2) diluent + testosterone pellet; (3) daily injections of $25 \mu \mathrm{g}$ melatonin; (4) daily injections of $25 \mu \mathrm{g}$ melatonin + testosterone pellet; and (5) daily injections of $25 \mu \mathrm{g}$ melatonin, a testosterone pellet and a melatonin pellet. The remaining 27 animals were moved to a room providing a short photoperiod (2L:22D; lights on 10:00-12:00 h) and divided into 3 treatment groups: (6) controls; (7) testosterone pellet; and (8) testosterone pellet and a melatonin pellet. Rats were killed between 09:00 and 12:00 h at the end of 12 weeks of treatment. The testes, accessory organs, pituitary, and body weights were recorded. Blood was collected in heparinized tubes. Plasma and pituitary samples were stored at $-70^{\circ} \mathrm{C}$ for hormone analysis. 


\section{Hormone analyses}

Luteinizing hormone (LH) and prolactin were evaluated in plasma and pituitary samples using reference preparation, standards and hormone for iodination provided by the National Institute of Arthritis, Digestive Diseases and Kidney (NIADDK). Thyrotrophin (TSH) was measured in plasma only. Thyroxine and triiodothyronine were determined by RIA using kits from Diagnostic Products (Los Angeles, CA). An inverse index of the fraction of thyroxine or triiodothyronine bound to plasma proteins was ascertained by determining the in-vitro proportional triiodothyronine uptake (Diagnostic Products kit) from plasma onto immobilized antibody. Free thyroxine and triiodothyronine indices were obtained by calculating the product of the respective total thyronine concentration and the triiodothyronine uptake value.

\section{Statistical analysis}

Data were analysed using a one-way analysis of variance and a Student-Newman-Keuls test for differences among multiple means.

\section{Results}

As shown in Fig. 1, daily afternoon injections of $25 \mu \mathrm{g}$ melatonin for 12 weeks had no effect on testicular weight of male rats kept in long photoperiod (Group 3); similarly, exposure of rats to a short photoperiod (Group 6) had no effect on gonadal weight. However, rats maintained in either photoperiod and implanted every 2 weeks with a testosterone pellet (Groups 2 and 7) showed a significant reduction in both absolute and relative testicular weight; this effect was significantly more pronounced in Group 7 (Fig. 1). The inhibitory effects of testosterone alone on both absolute and relative testicular weights were exacerbated by melatonin injections (Group 4). Subcutaneous 2-weekly implants of a beeswax pellet containing $1 \mathrm{mg}$ melatonin reversed the effects of melatonin injections on relative testicular weights (Group 5) and tended to reverse the effects of short photoperiod (Group 8) but this was not statistically significant. No significant differences in accessory organ weights were observed.

Figure 2 shows that testosterone implants significantly reduced pituitary LH values in Groups 2 and 7. Melatonin injections alone or in combination with melatonin pellets did not further exaggerate

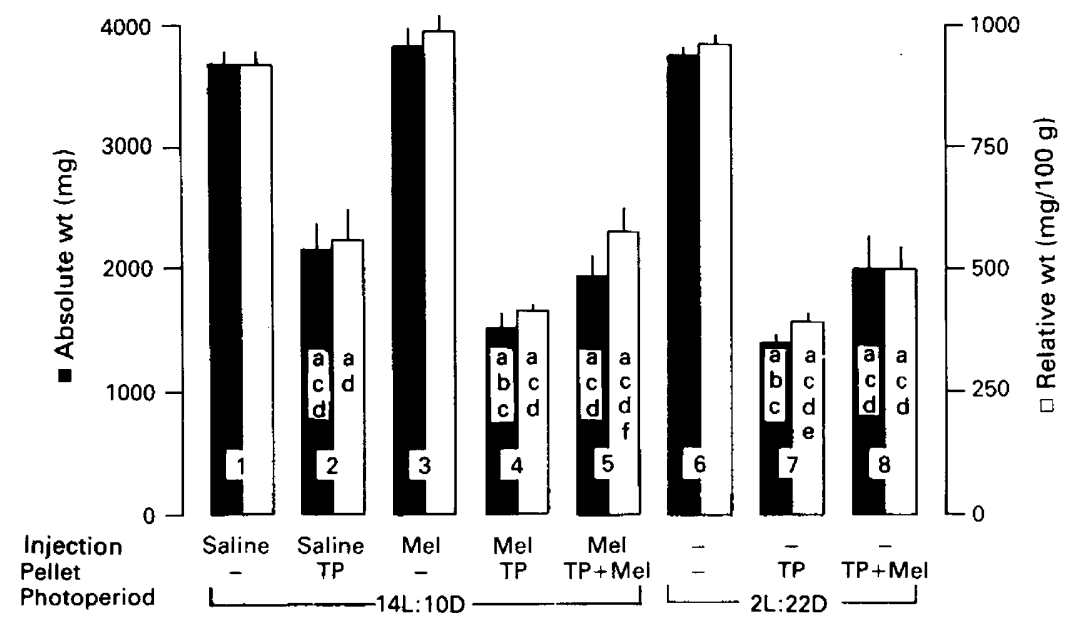

Fig. 1. Absolute and relative testes weights of rats maintained in long (14L:10D) or short (2L: 22D) photoperiod. Group numbers are shown at the bottom of each bar. Mel = melatonin; $\mathrm{TP}=$ testosterone propionate. Means \pm s.e.m. are indicated. a, $P<0.001$ vs Group $1 ; \mathrm{b}$, $P<0.025$ vs Group 2; c, $P<0.001$ vs Group 3; d, $P<0.001$ vs Group 6; e, $P<0.05$ vs Group 2; f, $P<0.025 v s$ Group 4. 


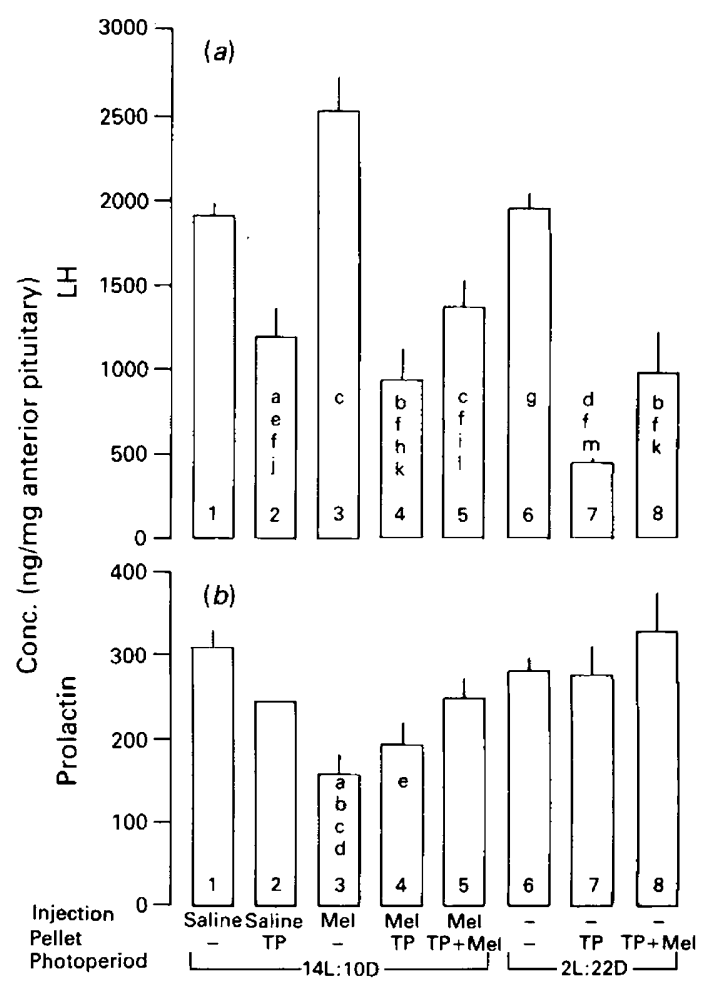

Fig. 2. Pituitary concentrations of (a) LH and (b) prolactin for rats in Groups $1-8(N=6-9$ rats/group). Group numbers are shown at the bottom of each bar. Mel = melatonin; TP = testosterone propionate. Values are means \pm s.e.m. In (a), a, $P<0.025, \mathrm{~b}, P<0.005, \mathrm{c}$, $P<0.05$ vs Group 1; d, $P<0.001$ vs Group 6; e or h, $P<0.001$ vs Group 3; f, $P<0.025$ vs Group 6; g, $P<0.025$ vs Group 3; i, $P<0.01$ vs Group 3; k, $P<0.05$ vs Group 7; $1, P<0.005$ vs Group 7; m, $P<0.001$ vs Groups $1 \&$ 3. In (b), a, $P<0.01$ vs Group 1; b, $P<0.05$ vs Group 6; c, $P<0.05$ vs Group 7; d, $P<0.005$ vs Group 8; e, $P<0.025$ vs Group 8.

the depression in pituitary LH due to testosterone pellets alone in rats in Groups 4 and 5; similarly, melatonin pellets did not reverse the depression in pituitary $\mathrm{LH}$ observed in rats in Group 8 . No significant differences in plasma LH values were found (Table 1).

Pituitary prolactin values (Fig. 2) were significantly reduced in rats in Group 3. No significant differences were observed in plasma prolactin concentrations (Table 1).

Body weights of rats in Group 4 were significantly depressed compared to the weights of animals in Groups 1, 3, 6 and 8 (Table 1). Absolute pituitary weights of rats in Group 3 were elevated compared to those of Group 7 , but when pituitary weight was expressed relative to body weight, these differences disappeared (Table 1).

Thyroxine concentrations as well as the free thyroxine index were unchanged by any combination of treatments (Table 1). Rats in Groups 3, 4 and 6 had the highest levels of triiodothyronine and free triiodothyronine index while these values were lowest in Group 8 rats (Table 1). Injections of melatonin (Group 3) caused a significant rise in plasma concentrations of TSH compared to Groups 2, 5, 6, 7 and 8 (Table 1). The ratio of thyroxine to triiodothyronine indicates that groups exposed to a short photoperiod had a higher ratio of these thyroid hormones compared to groups on long photoperiod (Fig. 3). 


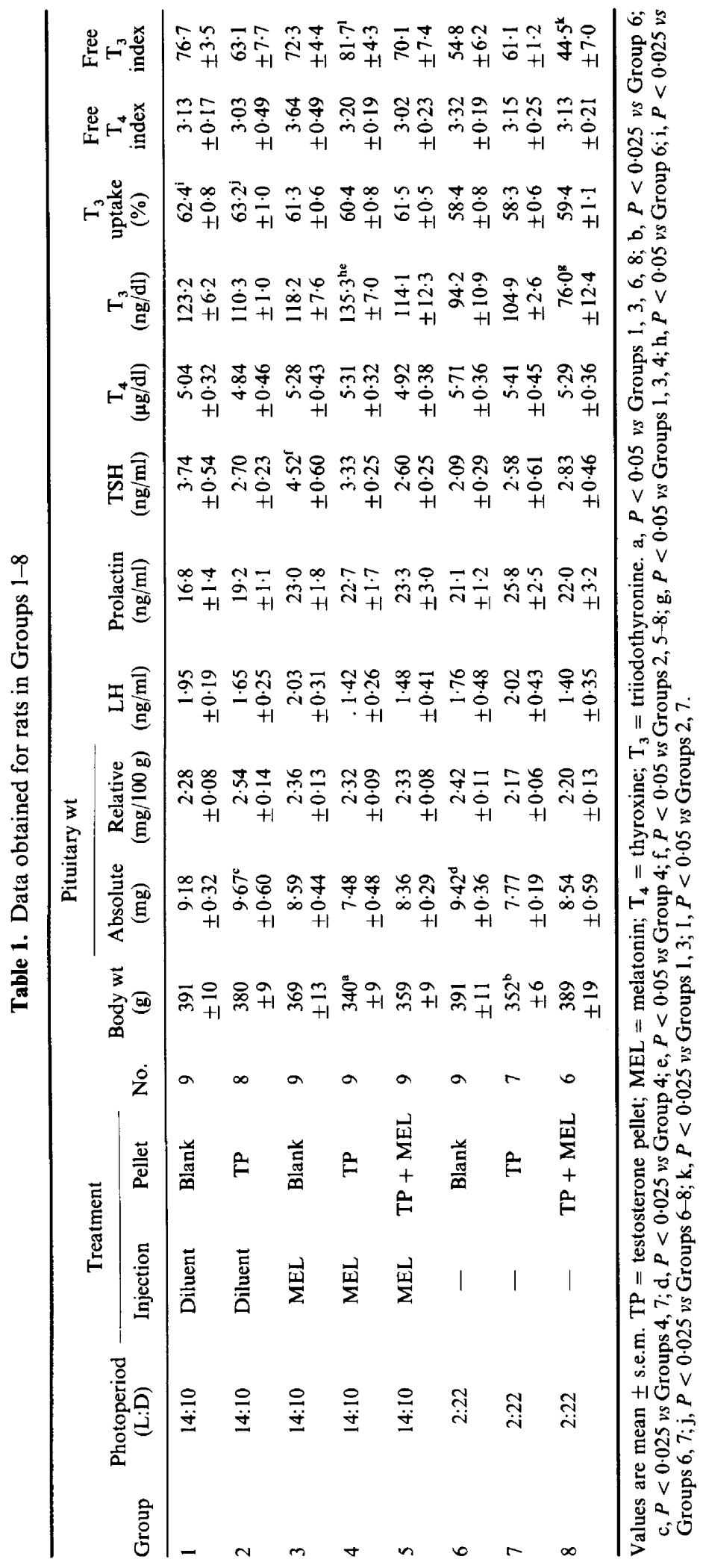




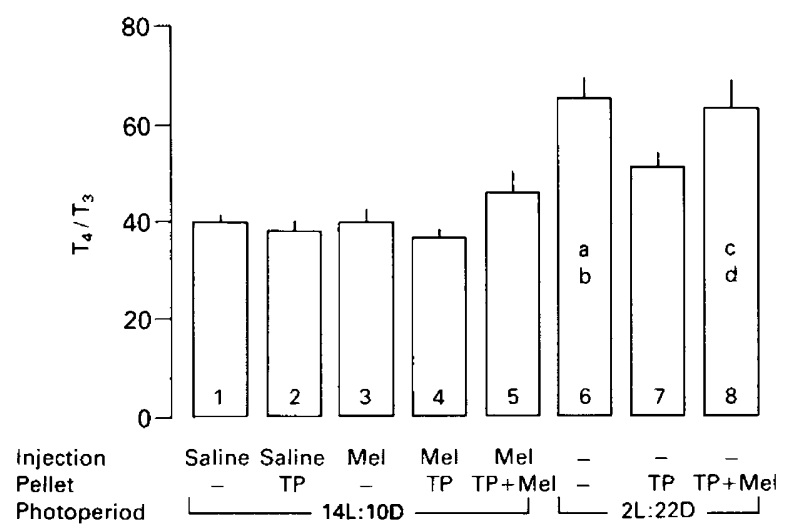

Fig. 3. Ratio of thyroxine $\left(T_{4}\right) /$ triiodothyronine $\left(T_{3}\right)$ in rats in long (14L:10D) or short (2L:22D) photoperiod. Group numbers are shown at the bottom of each bar. Mel = melatonin; TP = testosterone propionate. Values are means \pm s.e.m. a, $P<0.005$ vs Groups 1, 2, 3, 4; b, $P<0.025$ vs Group 5; c, $P<0.005$ vs Groups 1, 2, 3, 4; d, $P<0.025$ vs Group 5.

\section{Discussion}

Numerous studies of rats have shown that decreasing photic input in combination with one of several manipulations (underfeeding, anosmia, neonatal androgenization) can sensitize the neuroendocrinereproductive axis to the effects of short photoperiod or melatonin injections (Reiter et al., 1975a). In the present study, implanted testosterone pellets served to prime the animal so that the inhibitory actions of short photoperiod or melatonin injections on testis weight were unmasked, confirming that testosterone given in adulthood can be added to the growing list of pineal potentiating factors.

Chronic subcutaneous implants of Silastic capsules containing testosterone may suppress or maintain spermatogenesis and testicular weight depending upon the length and/or wall thickness of the capsule implanted (Ewing et al., 1973; Desjardins et al., 1973; Berndtson et al., 1974). In the present study, we confirmed the observations of Wallen \& Turek (1981) and Wallen et al. (1983) that testosterone pellets reduce gonadal weight and that the suppression is more prominent in rats kept in a short photoperiod. The mechanism involved may be similar to that in the hamster in which there is increased sensitivity of the neuroendocrine axis to testosterone feedback in animals maintained in short days; this phenomenon is known to be in part mediated by the pineal gland (Turek, 1979).

Melatonin injections into adult testosterone-treated rats in a long photoperiod exacerbated the inhibitory effect of testosterone alone on testicular weight. This observation suggests that melatonin is as effective as short photoperiod on the neuroendocrine-reproductive axis and may be the active agent in mediating the effects of short photoperiod. Similar results have been shown in immature rats in which daily afternoon injections of melatonin are known to evoke the same response as short photoperiod when coupled with these sensitizing factors in immature animals (Blask \& Nodelman, 1979, 1980; Blask et al., 1980). In terms of inhibiting the peripheral effects of testosterone, melatonin injections prevented the pinealectomy-induced enhanced growth response of the seminal vesicles to 10 days of androgen injection in immature castrated rats (Alonso et al., 1978); however, this inhibitory effect of melatonin was not observed in the present study in which exogenous androgen was administered to adult rats. We can therefore conclude that melatonin injections inhibit the weights of the reproductive organs in the immature (Lang, 1986) and adult rat if the animals are properly primed by certain predisposing environmental conditions or agents. 
Male rats treated with androgen pellets which suppress testicular weight typically demonstrate histological and endocrinological characteristics reminiscent of those observed after hypophysectomy in rodents (Berndtson et al., 1974; Ewing et al., 1973). Neither pituitary weight nor plasma LH was suppressed by the dose of testosterone used in the long photoperiod-exposed rats in the present study although pituitary LH was significantly depressed. However, when short photoperiod was added to testosterone treatment, rats exhibited a marked depression in pituitary weight and pituitary LH concentration, indicating that the neuroendocrine axis was made more sensitive to the negative feedback effects of testosterone by short photoperiod.

Previous studies of rats with pineal-mediated gonadal regression demonstrate that subcutaneous deposits of melatonin reversed the antigonadotrophic effects induced by the combination of blinding with anosmia (Chen \& Reiter, 1980) or neonatal androgenization (Banks \& Reiter, 1975). Melatonin pellets did reverse the effects of melatonin injections on relative testicular weight, supporting the hypothesis generated from data from rats and hamster that melatonin available as a pellet is counterantigonadotrophic. However, when administered to rats kept in a short photoperiod, the reversal was not quite statistically significant although inspection of Fig. 1 reveals that animals treated with short photoperiod + melatonin pellets (Group 8) had testicular weights more like those of Group 2 (testosterone-treated, long photoperiod-exposed animals) and Group 5 (melatonin-injected, testosterone pellet + melatonin pellets) than like those of Group 3 (testosterone-treated animals receiving melatonin injections) and/or Group 6 (short photoperiod exposure).

Previous studies of hamsters show a depression in thyroid hormones that parallels the ongoing gonadal atrophy due to short photoperiod or melatonin injections (Petterborg et al., 1984; Reiter et al., 1985). At the single timepoint measured in the present study, circulating thyroxine concentrations were not affected by the long-term treatment with short photoperiod or melatonin injections. This response is distinctly different from the pattern normally seen in the hamster but appears to be similar to that observed in other rodents (Petterborg et al., 1984). Rats kept in short photoperiod have a much higher thyroxine/triiodothyronine ratio than do those in the various treatment groups maintained in long photoperiod. Again, this is clearly a different pattern of response from that which is observed after melatonin or short photoperiod exposure in the hamster. Further, the present results indicate that a parallel response in the thyroid axis is not absolutely necessary for gonadal atrophy to occur in the rat.

Supported by NSF grant No. PCM 8410592 and Spanish-American Grant No. CCA 8309108.

\section{References}

Alonso, R., Prieto, L., Hernandez, C. \& Mas, M. (1978) Antiandrogenic effects of the pineal gland and melatonin in castrated rats and intact prepubertal male rats. $J$. Endocr. 79, 77-83.

Banks, A.F. \& Reiter, R.J. (1975) Melatonin inhibition of pineal antigonadotrophic activity in male rats. Hormone Res. 6, 351-356.

Berndtson, W.E., Desjardins, C. \& Ewing, L.L. (1974) Inhibition and maintenance of spermatogenesis in rats implanted with polydimethylsiloxane capsules containing various androgens. J. Endocr. 62, 125-135.

Blask, D.E. \& Nodelman, J.L. (1979) Antigonadotrophic and prolactin-inhibitory effects of melatonin in anosmic male rats. Neuroendocrinology 29, 406-412.

Blask, D.E. \& Nodelman, J.L. (1980) An interaction between the pineal gland and olfactory deprivation in potentiating the effects of melatonin on gonads, accessory sex organs, and prolactin in male rats. $J$. Neurosci. Res. 5, 129-136.
Blask, D.E., Nodelman, J.L., Leadem, C.A. \& Richardson, B.A. (1980) Influence of exogenously administered melatonin on the reproductive system and prolactin levels in underfed male rats. Biol. Reprod. 22, $507-512$.

Chen, H.J. \& Reiter, R.J. (1980) Influence of subcutaneous deposits of melatonin on the antigonadotrophic effects of blinding and anosmia in male rats. Neuroendocrinology 30, 169-173.

Desjardins, C., Ewing, L.L. \& Irby, D.C. (1973) Response of the rabbit seminiferous epithelium to testosterone administered via polydimethylsiloxane capsules. Endocrinology 93, 450-460.

Ewing, L.L., Stratton, L.G. \& Desjardins, C. (1973) Effect of testosterone polydimethylsiloxane implants upon sperm production, libido and accessory sex organ function in rabbits. $J$. Reprod. Fert. 35, 245-253.

Lang, U. (1986) Melatonin and puberty. Pineal Res. Rev. 4, 199-243. 
Petterborg, L.J., Vaughan, M.K., Johnson, L.Y., Champney, T.H. \& Reiter, R.J. (1984) Modification of testicular and thyroid function by chronic exposure to short photoperiod: a comparison in four rodent species. Comp. Biochem. Physiol. 78A, 31-34.

Reiter, R.J., Vaughan, M.K., Blask, D.E. \& Johnson, L.Y. (1974) Melatonin: its inhibition of pineal antigonadotrophic activity in male hamsters. Science, N.Y. 185, 1169-1170.

Reiter, R.J., Vaughan, M.K., Vaughan, G.M., Sorrentino, S. \& Donofrio, R.J. (1975a) The pineal gland as an organ of internal secretion. In Frontiers of Pineal Physiology, pp. 54-174. Ed. M. D. Altschule. MIT Press, Cambridge.

Reiter, R.J., Blask, D.E. \& Vaughan, M.K. (1975b) A counter-antigonadotrophic effect on melatonin in male rats. Neuroendocrinology 19, 72-80.

Reiter, R.J., Vaughan, M.K., King, T.S. \& Karasek, M. (1985) The mammalian pineal gland: pharmacologic regulation and physiologic consequences. In Handbook of Pharmacologic Methodologies for the Study of the Neuroendocrine System, pp. 331-384. Eds R. W. Steger \& A. Johns. CRC Press, Boca Raton.
Turek, F.W. (1979) Role of the pineal gland in photoperiod-induced changes in hypothalamicpituitary sensitivity to testosterone feedback in castrated male hamsters. Endocrinology 104, 636-640.

Vaughan, M.K., Richardson, B.A., Petterborg, L.J., Vaughan, G.M. \& Reiter, R.J. (1985) Pineal indoles and analogues - effects on thyroxine in the Syrian hamster. Adv. Biosci. 53, 251-256.

Wallen, E.P. \& Turek, F.W. (1981) Photoperiodicity in the male albino laboratory rat. Nature, Lond. 289, 402-404.

Wallen, E.P., Reyes, S., Rynders, S., DeRosch, J., Georgeson, J. \& Losee-Olson, S. (1983) Photoperiodic responses in the male albino laboratory rat. Biol. Reprod. Suppl. 1, 125, Abstr. 186.

Received 26 May 1987 